S.A. Sajjadi, MRCP

K. Patterson, FMedSci

R.J. Arnold

P.C. Watson, PhD

P.J. Nestor, FRACP,

$\mathrm{PhD}$

Correspondence \& reprint requests to Dr. Nestor: pjn23@hermes.cam.ac.uk
Supplemental data at www.neurology.org

Supplemental Data

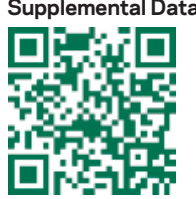

\title{
Primary progressive aphasia
}

\author{
A tale of two syndromes and the rest
}

\section{ABSTRACT}

Objective: Primary progressive aphasia (PPA) has been proposed to comprise 3 discrete clinical subtypes: semantic, agrammatic/nonfluent, and logopenic. Recent consensus recommendations suggest a diagnostic framework based primarily on clinical and neuropsychological findings to classify these variants. Our objective was to evaluate the extent to which patients with PPA would conform to the proposed tripartite system and whether the clustering pattern of elements of the linguistic profile suggests discrete clinical syndromes.

Methods: A total of 46 patients with PPA were prospectively recruited to the Cambridge Longitudinal Study of PPA. Sufficient data were collected to assess all consensus-proposed diagnostic domains. By comparing patients' performances against those of 30 age- and education-matched healthy volunteers, $z$ scores were calculated, and values of 1.5 SDs outside control participants' means were considered abnormal. Raw test scores were used to undertake a principal factor analysis to identify the clustering pattern of individual measures.

Results: Of the patients, $28.3 \%, 26.1 \%$, and $4.3 \%$ fitted semantic, nonfluent/agrammatic, and logopenic categories respectively, and $41.3 \%$ did not fulfill the diagnostic recommendations for any of the 3 proposed variants. There was no significant between-group difference in age, education, or disease duration. Furthermore, the outcome of the factor analysis was in keeping with discrete semantic and nonfluent/agrammatic syndromes but did not support a logopenic variant.

Conclusion: Taken together, the results of this prospective data-driven study suggest that although a substantial proportion of patients with PPA have neither the semantic nor the nonfluent variants, they do not necessarily conform to a discrete logopenic variant. Neurology ${ }^{\circledR}$ 2012;78:1670-1677

\section{GLOSSARY}

IvPPA = logopenic variant of primary progressive aphasia; nfvPPA = nonfluent/agrammatic variant of primary progressive aphasia; PPA = primary progressive aphasia; svPPA = semantic variant of primary progressive aphasia.

Primary progressive aphasia (PPA) is a collective term for a group of clinically and pathologically heterogeneous disorders in which a neurodegenerative disease presents with language impairment as the most salient feature. ${ }^{1}$ The current clinical formulation posits 3 variants: semantic variant (svPPA, also known as semantic dementia), nonfluent/agrammatic variant (nfvPPA), and logopenic variant (lvPPA). ${ }^{2-4}$ Recently, diagnostic recommendations (table 1) have been proposed to enable identification of these 3 most commonly reported clinical presentations of PPA. ${ }^{5}$ It remains unclear, however, whether there are only 3 definable syndromes or indeed whether all 3 of these proposed categories are sufficiently discrete and consistent to justify them being delineated as specific syndromes.

A particular advance with these proposed recommendations was the stipulation of specific types of tests to assess certain language features. Many of the recommendations, however, still rely on evaluating connected speech. Despite publication of a few formal analyses, ${ }^{6,7}$ connected speech is typically not quantified and, hence, at risk of inconsistent evaluation or bias. The Cambridge Longitudinal Study of PPA was established for prospective evaluation of the phe-

From the Neurology Unit (S.A.S., K.P., R.J.A., P.J.N.), University of Cambridge, Herchel Smith Building for Brain and Mind Sciences, Cambridge, UK; and Methods Group (P.C.W.), MRC Cognition and Brain Sciences, Cambridge, UK.

Study funding: Supported by the Donald Forrester Trust research grant to S.A.S. and NIHR Cambridge Biomedical Research Centre

Go to Neurology.org for full disclosures. Disclosures deemed relevant by the authors, if any, are provided at the end of this article. 
Table 1 Proposed recommendations for clinical diagnosis of PPA variants

\begin{tabular}{|c|c|c|c|}
\hline & $\begin{array}{l}\text { nfvPPA: At least one core and } \\
\text { two-thirds of other features }\end{array}$ & $\begin{array}{l}\text { svPPA: Both core and } \\
\text { three-fourths of other } \\
\text { features }\end{array}$ & $\begin{array}{l}\text { IvPPA: Both core and } \\
\text { three-fourths of other } \\
\text { features }\end{array}$ \\
\hline \multirow[t]{2}{*}{ Core features } & $\begin{array}{l}\text { Agrammatism in language } \\
\text { production }\end{array}$ & Impaired confrontation naming & $\begin{array}{l}\text { Impaired single word retrieval } \\
\text { in speech and naming }\end{array}$ \\
\hline & $\begin{array}{l}\text { Effortful halting speech with } \\
\text { inconsistent speech sound errors } \\
\text { and distortions }\end{array}$ & $\begin{array}{l}\text { Impaired single-word } \\
\text { comprehension }\end{array}$ & $\begin{array}{l}\text { Impaired repetition of } \\
\text { sentences and phrases }\end{array}$ \\
\hline \multirow[t]{4}{*}{$\begin{array}{l}\text { Other diagnostic } \\
\text { features }\end{array}$} & $\begin{array}{l}\text { Impaired comprehension of } \\
\text { syntactically complex sentences }\end{array}$ & Impaired object knowledge & $\begin{array}{l}\text { Phonologic errors in speech } \\
\text { and naming }\end{array}$ \\
\hline & Spared single word comprehension & Surface dyslexia or dysgraphia & $\begin{array}{l}\text { Spared single word } \\
\text { comprehension and object } \\
\text { knowledge }\end{array}$ \\
\hline & Spared object knowledge & Spared repetition & Spared motor speech \\
\hline & & Spared speech production & Absence of frank agrammatism \\
\hline
\end{tabular}

Abbreviations: IvPPA = logopenic variant of primary progressive aphasia; nfvPPA = nonfluent/agrammatic variant of primary progressive aphasia; svPPA = semantic variant of primary progressive aphasia.

nomenology of PPA, using a detailed neuropsychological battery and formal analysis of connected speech. In this study, 46 consecutively recruited patients were tested to examine the extent to which patients would conform to the proposed tripartite system. In addition, a purely data-driven analysis was undertaken to identify the elements of linguistic impairment that cluster together, thus using empirical evidence to test for the existence of discrete syndromic variants.

METHODS Participants. A total of 46 patients with a clinical diagnosis of PPA were prospectively recruited over a 2 year period (2009-2011) from the memory clinics held at Addenbrooke's Hospital, University of Cambridge, UK. All patients met the basic criteria for PPA. ${ }^{5}$ Nondegenerative pathologies were excluded using MRI, except in 3 patients who had CT because MRI was contraindicated. Previous experience had shown that cognitive screening tests such as the Mini-Mental State Examination, for which responses often require speaking, could be misleading in assessment of functional abilities in patients with prominent language problems. ${ }^{8,9}$ We designed an informant-filled questionnaire on activities of daily living to assess patients' ability to cope with language-independent day-today activities. In addition, prospective participants were excluded if 1) English was not their first language (excluded 1 patient) or 2) their language was so impaired that they could not provide speech samples of the length and quality required for meaningful analysis (excluded 1 patient). Spouses/partners of patients were recruited as control subjects to be matched for general demographic factors, especially age and education. All were free of cognitive symptoms and neurologic or psychiatric illnesses and performed normally on Addenbrooke's Cognitive Examination-Revised. ${ }^{10}$

Standard protocol approvals, registrations, and patient consents. Written informed consent was obtained from the participants and, where appropriate, their next of kin. The study was approved by the regional ethics committee.
Neuropsychological battery. All subjects with PPA and control participants performed a similar set of general neuropsychological tests (table 2) and an extensive battery of language-specific tests that, along with the outcome of their connected speech analysis, were explicitly chosen to evaluate all consensus-proposed language markers. Connected speech was elicited in 2 ways: 1) by description of the picture (a busy domestic scene) from the Dutch version of the Comprehensive Aphasia Test ${ }^{11}$ and 2) by semistructured interviews. To provide an objective measure of the patients' performance relative to that of healthy participants and to binarize the individual test scores to normal or abnormal, which was required for the first part of the analyses, standard scores were calculated. To examine the degree to which the patients could be classified using the recently proposed recommendations, their standard scores were used to assess the presence or absence of the diagnostic features for each of the 3 proposed variants. Inspired by criteria for minimal cognitive impairment, ${ }^{12}$ a threshold of $1.5 \mathrm{SDs}$ worse than the control participants' mean was set as the boundary of normal. Data were also analyzed using a $95 \%$ confidence interval (1.96 SDs) to see whether this stricter threshold would be more specific in assigning patients to syndromic groups. Raw scores were used for principal factor analysis.

Based on the consensus recommendations, the following language features were assessed: agrammatism in language production; motor speech impairment; comprehension of syntactically complex structures; basic syntactic production skills; word and sentence repetition; confrontation naming; single word comprehension; object knowledge; surface dyslexia; and frequency of phonologic errors in connected speech. Methodologic details for the assessment of these language markers are presented in appendix e-1 on the Neurology ${ }^{\circledR}$ Web site at www.neurology.org.

Statistical considerations. Predictive Analytics SoftWare (PASW) version 18 was used for statistical analysis of the data. $z$ scores were calculated using the transform function in PASW. Oneway analysis of variance or its nonparametric equivalent, the Kruskal-Wallis test, was used to compare the demographic and general neuropsychological markers in different groups of patients and control participants. A 2-tailed $p$ value of 0.05 was considered significant. We also undertook principal factor analysis with orthogonal varimax rotation on the 14 speech features listed in table 3 to identify the clustering pattern of individual measures. Factors with eigenvalues over the Keiser criterion of 1 were retained. ${ }^{13}$ 
Table 2 Frequency of syndromic variants and comparison of demographic and general neuropsychological markers

\begin{tabular}{|c|c|c|c|c|c|c|}
\hline & nfvPPA & svPPA & IvPPA & Mixed PPA & Control & $\begin{array}{l}\text { Omnibus } \\
\text { significance: } \\
\text { p }(<0.05)\end{array}$ \\
\hline No. (\%) & $12(26.1)$ & $13(28.3)$ & $2(4.3)$ & 19 (41.3) & 30 & \\
\hline \multicolumn{7}{|l|}{$\begin{array}{l}\text { Demographic } \\
\text { markers, } \\
\text { mean (range) }\end{array}$} \\
\hline Age at test, $y$ & $68(53-77)$ & 68 (61-79) & $71(68-74)$ & $70(60-83)$ & $67.5(51-80)$ & NS \\
\hline $\begin{array}{l}\text { Disease duration, } \\
\text { y }\end{array}$ & $3.5(2-5)$ & $4.4(2.5-7)$ & $2(1.5-2.5)$ & $3.9(2-7)$ & NA & NS \\
\hline Education, y & $13(10-20)$ & $14(10-19)$ & $11(9-13)$ & $11(9-16)$ & $12.8(10-20)$ & NS \\
\hline ADL-Q & $0.73(0-4)$ & $1(0-5)$ & $0.5(0-1)$ & $1.3(0-6)$ & NA & NS \\
\hline \multicolumn{7}{|l|}{$\begin{array}{l}\text { General } \\
\text { neuropsychological } \\
\text { measures, } \\
\text { mean (range) }\end{array}$} \\
\hline MMSE (30) ${ }^{a}$ & $24.6(15-28)^{\star \star *}$ & $23(18-27)^{* * *}$ & $25.5(25-26)^{\star *}$ & $17.9(10-28)^{* * *, b, c, d}$ & $29.2(28-30)$ & $<0.001$ \\
\hline ACE-R (100) & $74.1(44-89)^{\star \star *}$ & $52(36-87)^{\star \star *, e}$ & $72.0(68-76)^{\star \star *}$ & $46.5(28-69)^{\star \star *, b, f, g}$ & 94.4 (88-99) & $<0.001$ \\
\hline $\begin{array}{l}\text { Forward digit } \\
\text { span (8) }\end{array}$ & $4.5(3-7)^{* \star *, c}$ & $6.3(4-8)$ & $5.1(4-6)^{*, f}$ & $4.4(3-8)^{* *+, h}$ & $6.7(5-8)$ & $<0.001$ \\
\hline $\begin{array}{l}\text { Backward digit } \\
\text { span (7) }\end{array}$ & $3.7(0-4)^{* * *}$ & $4.2(3-7)$ & $4.0(3-5)^{*}$ & $3.0(0-6)^{* * *, c}$ & $5.1(3-8)$ & $<0.001$ \\
\hline $\begin{array}{l}\text { Letter fluency, } \\
\text { words/min }\end{array}$ & $5.3(1-11)^{* * * *}$ & $6.0(1-11)^{* * * *}$ & $6.5(6-7)^{* *}$ & $4.8(1-11)^{* * *}$ & $14.4(4-21)$ & $<0.001$ \\
\hline $\operatorname{VOSP}(10)^{i}$ & $8.2(4-10)^{*, f}$ & $9.8(9-10)$ & $7.5(5-10)^{*}$ & $6.6(2-10)^{* *, f}$ & $9.3(8-10)$ & $<0.001$ \\
\hline $\begin{array}{l}\text { Rey figure } \\
\text { copy (36) }\end{array}$ & $26.5(2.5-36)^{* * *, f}$ & $33(30-36)$ & $20.5(19-22)^{* \star *, c}$ & $21.1(0.5-35)^{n+*, h}$ & 34.6 (29-36) & $<0.001$ \\
\hline $\begin{array}{l}\text { Rey figure } \\
\text { recall (36) }\end{array}$ & $10.7(0-23)^{* *}$ & $10(0-25)^{*}$ & $5.5(2-9)^{* *}$ & $7.4(0-21)^{* * * *}$ & $18.2(0-29)$ & $<0.001$ \\
\hline Trails-A (time), $s^{i}$ & $104.5(47-394)^{* *+\ldots, f}$ & $49.2(21-98)$ & $93(69-117)^{* *}$ & $106.6(48-445)^{* * *, f}$ & $37.6(22-62)$ & $<0.001$ \\
\hline
\end{tabular}

Abbreviations: $A C E-R=$ Addenbrooke's Cognitive Examination-Revised; $A D L-Q=$ activities of daily living questionnaire; IVPPA = logopenic variant of PPA; MMSE = Mini-Mental State Examination; NA = not applicable; nfvPPA = nonfluent/ agrammatic variant of PPA; NS = nonsignificant; svPPA = semantic variant of PPA; VOSP = Visual Object and Perception Battery (cube analysis).

${ }^{*} p<0.05$ compared with the control population.

${ }^{* *} p<0.01$ compared with the control population.

${ }^{* * *} p<0.001$ compared with the control population.

a Numbers in parentheses indicate the maximum score for each test.

${ }^{\mathrm{b}} p<0.001$ compared with nfvPPA.

${ }^{c} p<0.01$ compared with svPPA.

${ }^{d} p<0.001$ compared with IvPPA.

e $p<0.01$ compared with nfvPPA.

${ }^{f} p<0.05$ compared with svPPA.

${ }^{g} p<0.01$ compared with IvPPA.

${ }^{h} p<0.001$ compared with svPPA.

i Nonparametric test.

RESULTS Assessment of the proposed recommendations. Every patient was assessed against the diagnostic recommendations for each of the 3 proposed variants. With a $z$ score threshold of 1.5 , the score for none of the patients was too mild to meet the minimum diagnostic criteria for one of the syndromic variants. The frequencies of the syndromic variants are summarized in table 2 along with their demographic and general neuropsychological features.

Of the patients, 27 (59\%) fitted 1 of the 3 categories, but 19 patients (41\%) either fulfilled the diagnostic criteria for more than one variant or were too impaired to fulfill the criteria for any variant (see table e-1 for an example for one patient). These latter 19 patients were labeled mixed. Repeating this procedure using the more stringent threshold of $\geq 1.96$ $\mathrm{SDs}$ from the control mean led to a change in syndromic classification for only one patient, from nonfluent/agrammatic to mild/impaired on a single measure but did not affect the mixed group membership. Table 4 provides the neuropsychological and speech data for every patient in the mixed group. There was no statistically significant difference between the 4 patient groups (svPPA, nfvPPA, lvPPA, 


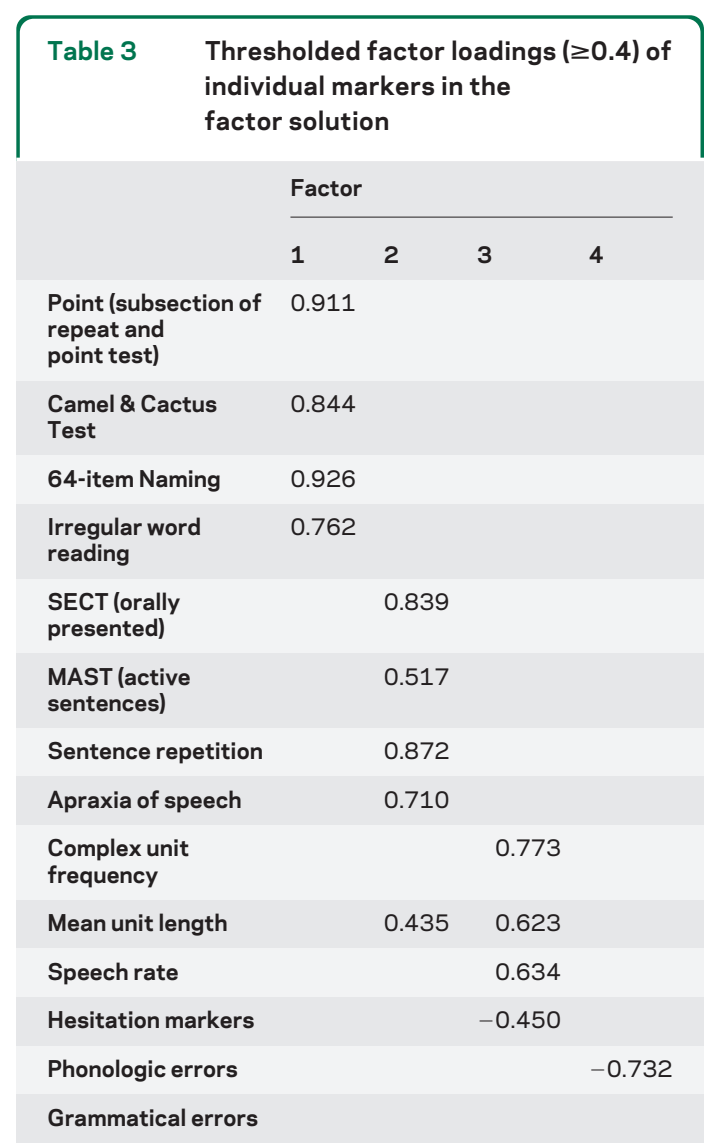

Abbreviations: $\mathrm{MAST}=$ make a sentence test $; \mathrm{SECT}=$ sentence comprehension test.

and mixed PPA) and control participants in terms of their demographic markers.

Principal factor analysis. The Keiser-Myer-Olkin measure verified the sampling adequacy for the analysis (Keiser-Myer-Olkin $=0.68$, significance $<0.001)$, and the rotation converged in 6 iterations. The correlation matrix confirmed satisfactory correlation coefficients for all but the grammatical error score in connected speech, which was not significantly correlated with any of the other markers. Four factors with eigenvalues more than 1 accounted for $64 \%$ of the variance in the data. Examination of the Scree plot (figure) revealed a large step down in eigenvalues from factor 2 to factor 3 . The first 2 factors accounted for $42 \%$ of the variance. Table 3 shows thresholded factor loadings of individual markers in the factor solution (for an unthresholded version of the table, see table e-2). Scrutiny of the rotated factor matrix indicated that factor 1 clustered semantic measures, factor 2 clustered measures of grammatical competence, sentence repetition, apraxia of speech and, with a weaker factor loading, mean length of utterance, and factor 3 clustered a number of connected speech markers. The fourth factor comprised only one measure (phonologic errors).
DISCUSSION Publication of the recommendations for classification of PPA has been a necessary first step toward development of an empirical and unbiased categorization of the patients to clinically meaningful groups. Application of the recommendations to prospective cohorts such as this one provides an opportunity for evolution of these recommendations.

By applying the proposed measures for delineating the syndromic variants of PPA to this consecutively recruited cohort of 46 cases, $26.1 \%$ fulfilled the criteria for nfvPPA and $28.3 \%$ for svPPA but only $4.3 \%$ for lvPPA. A substantial $41.3 \%$ could only be classified as mixed PPA in that their deficits either extended beyond a single syndromic variant domain (i.e., they violated the stipulation that for a given syndromic variant a certain feature had to be spared) or they met the criteria for more than one variant. The possibility that this large group of mixed cases might be attributable to a longer duration of disease was not supported by the demographic data: the mixed group had disease duration similar to that of the other groups. The patients with mixed PPA fared worse on general neuropsychological measures at a group level, but this observation should be interpreted with caution. Because their aphasia was also more diffuse, it may be that it has a more pervasive effect on general cognition as has been noted previously. ${ }^{8,14}$ It is important to highlight the possibility that performance on general cognitive measures was not systematically worse in all patients with mixed aphasia. Finally, it is worth noting that, from a pathologic point of view, such performance is not in itself evidence for a different pathologic grouping: in a previous study, nonlinguistic measures were inconsistently affected across pathologic substrates for PPA. ${ }^{15}$

Of note, the authors of the recent recommendations speculated that there may be instances in which language impairment is so mild that the patient fails to meet any criteria (i.e., by only being impaired on a single measure) $)^{5,16}$; in this prospective sample, no patients fell into this category with a cutoff of $1.5 \mathrm{SDs}$ and only one patient at a threshold of 1.96.

One could argue that strict application of cutoff scores to define the impairments that were set out in the consensus article might be too rigid to recognize the nuances of the proposed syndromic categories. It is therefore notable that principle axis factoring, involving no prior assumptions as to the existence of predetermined syndromic variants, yielded a pattern identical to the application of the consensus recommendations: 2 clear syndromes and a residual miscellany. The first factor clustered single word comprehension, nonverbal associative knowledge, picture naming, and irregular word reading. All of 
Table 4 Individual standard and percentage corrected scores for neuropsychological and speech measures in the mixed group ${ }^{a}$

\begin{tabular}{|c|c|c|c|c|c|c|c|c|c|c|c|}
\hline \multirow[b]{2}{*}{ Patient } & \multicolumn{11}{|c|}{ Domain $^{\mathrm{a}}$ and test } \\
\hline & $\begin{array}{l}\text { Object } \\
\text { knowledge: } \\
\text { CCT, \% }\end{array}$ & $\begin{array}{l}\text { Single } \\
\text { word } \\
\text { comp: } \\
\text { Point, \% }\end{array}$ & $\begin{array}{l}\text { Reading: } \\
\text { Irregular } \\
\text { word } \\
\text { reading, \% }\end{array}$ & $\begin{array}{l}\text { Confrontation } \\
\text { naming: } \\
64-\text {-item } \\
\text { naming, } \%\end{array}$ & $\begin{array}{l}\text { Repetition: } \\
\text { Sentence } \\
\text { repetition, \% }\end{array}$ & $\begin{array}{l}\text { Sentence } \\
\text { comp: } \\
\text { SECT, \% }\end{array}$ & $\begin{array}{l}\text { MAST- } \\
\text { Active } \\
\text { sentence, \% }\end{array}$ & $\begin{array}{l}\text { Agrammatism, } \\
\text { composite } \\
\text { z-scores }\end{array}$ & $\begin{array}{l}\text { Phonologic } \\
\text { errors, } \\
\text { z-score }\end{array}$ & $\begin{array}{l}\text { Hesitation } \\
\text { markers, } \\
\text { z-score }\end{array}$ & $\begin{array}{l}\text { Apraxia } \\
\text { of } \\
\text { speech }\end{array}$ \\
\hline 1 & $44^{b}$ & $80^{c}$ & $40^{b}$ & $81^{c}$ & $25^{b}$ & $40^{b}$ & $67^{b}$ & $2.61^{b}$ & $3.9^{b}$ & $1.8^{\mathrm{b}}$ & + \\
\hline 4 & $30^{b}$ & $40^{b}$ & 100 & $48^{b}$ & $17^{\mathrm{b}}$ & $0^{b}$ & $0^{\mathrm{b}}$ & $3.20^{b}$ & $10.3^{b}$ & -1.0 & + \\
\hline 5 & $62^{b}$ & $70^{\mathrm{b}}$ & $60^{b}$ & $2^{\mathrm{b}}$ & $67^{b}$ & $20^{b}$ & $0^{b}$ & 0.49 & $4.5^{\mathrm{b}}$ & $4.9^{b}$ & + \\
\hline 6 & $\mathrm{O}^{\mathrm{b}}$ & $40^{\mathrm{b}}$ & $60^{b}$ & $86^{c}$ & $58^{b}$ & $0^{b}$ & $0^{b}$ & $2.66^{b}$ & $18.1^{\mathrm{b}}$ & -1.0 & + \\
\hline 7 & 90 & 100 & 100 & $87^{c}$ & $25^{\mathrm{b}}$ & $32^{b}$ & $33^{b}$ & $2.29^{b}$ & $4.4^{\mathrm{b}}$ & $1.5^{\mathrm{b}}$ & - \\
\hline 11 & $55^{b}$ & $70^{b}$ & 100 & $22^{\mathrm{b}}$ & $75^{b}$ & $52^{b}$ & $50^{b}$ & $2.40^{b}$ & $23.3^{b}$ & $4.2^{b}$ & - \\
\hline 12 & $19^{b}$ & $40^{b}$ & 20 & $58^{b}$ & $17^{\mathrm{b}}$ & $16^{b}$ & $67^{b}$ & $2.09^{b}$ & -0.4 & $5.7^{b}$ & + \\
\hline 13 & $67^{b}$ & $60^{b}$ & 20 & $89^{c}$ & $8^{b}$ & $16^{b}$ & $0^{b}$ & $3.30^{b}$ & $12.4^{\mathrm{bb}}$ & $1.8^{\mathrm{b}}$ & + \\
\hline 14 & 86 & 100 & $80^{c}$ & $67^{b}$ & $17^{b}$ & $32^{b}$ & $17^{b}$ & $1.95^{b}$ & -0.4 & $1.9^{b}$ & + \\
\hline 15 & $55^{\mathrm{b}}$ & $50^{b}$ & 100 & $25^{\mathrm{b}}$ & $25^{\mathrm{b}}$ & $0^{\mathrm{b}}$ & $0^{b}$ & $2.47^{b}$ & $6.6^{b}$ & 0.5 & + \\
\hline 16 & $37^{b}$ & $50^{b}$ & 20 & $0^{b}$ & 92 & $60^{b}$ & $17^{b}$ & $2.19^{b}$ & -0.4 & -0.2 & - \\
\hline 17 & $36^{b}$ & $20^{\mathrm{b}}$ & 0 & $3^{b}$ & $0^{b}$ & $0^{b}$ & $0^{b}$ & 1.01 & $10.5^{b}$ & -0.3 & + \\
\hline 18 & $28^{b}$ & $20^{\mathrm{b}}$ & 0 & $27^{b}$ & $4^{b}$ & $0^{b}$ & $0^{b}$ & 1.19 & $11.4^{\mathrm{b}}$ & -0.1 & + \\
\hline 19 & $62^{b}$ & $60^{b}$ & 60 & $81^{c}$ & $83^{b}$ & $52^{b}$ & $67^{b}$ & 2.61 & -0.4 & -1.0 & - \\
\hline
\end{tabular}

Abbreviations: $\mathrm{CCT}=$ Camel and Cactus Test; comp = comprehension; MAST = Make A Sentence Test; SECT = Sentence Comprehension Test.

a Chosen as indicated in the recommendation article.

b Values outside $95 \%$ of the control group mean (z-scores $>1.96)$.

${ }^{c}$ Values indicate $1.5<z$-score $<1.96$.

these variables are classically associated with the syndrome of semantic dementia, indicating that a semantic variant of PPA is readily identifiable. The second factor, which was only slightly less significant than the semantic factor, clustered grammatical comprehension, sentence repetition, apraxia of speech, grammatical production, and mean length of utterance. This factor, therefore, appears to correspond to nfvPPA. Note, however, that impaired sentence repetition aligned with this factor, whereas it has been proposed to be a cardinal feature of lvPPA.

A binary rating of apraxia of speech was included in the analysis because it was listed as one of the key features in the recent classification article. ${ }^{5}$ It should be noted, however, that the definition of apraxia of speech remains a thorny issue because, at present, quantifiable measures for this feature are lacking. In this study, apraxia of speech was judged by 2 raters, blind to neuropsychological and clinical information, listening for "effortful halting speech with inconsistent speech sound errors and distortions" in recordings of connected speech and long word repe- tition. Although overall interrater agreement was fairly high (93\%), this was in large part because it was easy to decide on the absence of apraxia of speech in many of the patients. Making a positive diagnosis of apraxia of speech was more challenging. To date, the literature has largely defined apraxia of speech by clinical opinion, often citing that the rating was made by an expert. ${ }^{17-20}$ Without tangible criteria, however, this approach raises the question of whether one expert means the same thing as another. This problem is exemplified by the lack of agreement in how different groups have defined apraxia of speech. ${ }^{21-25}$ Moreover, even if interrater reliability between experts could be demonstrated, this may not be especially useful in cognitive neurology clinics in which such expertise will be variable. The present results suggest that, until validated measures are available, it might be better to abandon apraxia of speech as a classifying feature in PPA, given that the principle axis factoring strongly aligned this feature to agrammatism. Furthermore, removing apraxia of speech had no im- 


\section{Figure Scree plot showing the relative significance of different factors}

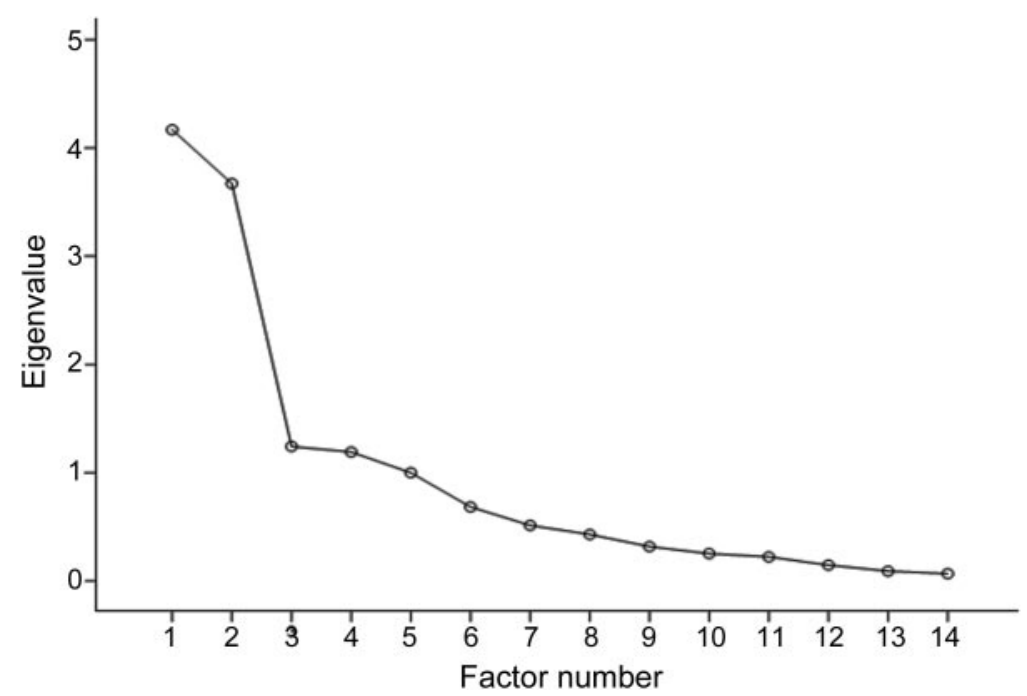

Note the significant reduction in eigenvalue from factor 2 to factor 3 .

pact on the clustering of the remaining markers in the factor matrix (table e-3).

After the semantic and agrammatic factors, there was a marked step down in significance for the remaining factors although 2 met the significance criterion of an eigenvalue $\geq 1$. The third axis included a number of connected speech measures comprising speech rate, frequency of complex grammatical structures, mean length of utterance (with a higher factor loading compared to the second axis), and frequency of hesitation markers. The fourth axis only included phonologic errors. As shown in the Scree plot (figure), the factors did not suggest evidence for a third discrete syndrome that stood out from the group. Furthermore, consistent with the application of clinical recommendations, which identified only 2 patients as meeting the proposed criteria for lvPPA, factors 3 and 4 did not resemble the description of logopenic aphasia. The proposed core features of lvPPA are impaired word retrieval (perhaps manifested by increased hesitation, i.e., word-finding pauses) and impaired sentence repetition, but these 2 features did not cluster together, with hesitations located in the third axis, whereas impaired sentence repetition aligned, with markers of agrammatism/ apraxia of speech, to the second factor. It is also noteworthy that, although the criteria for lvPPA merely specify impaired sentence repetition with no further qualifications, we did not penalize apraxic errors in our sentence repetition task and the scoring criteria were weighted toward detection of impairments in maintaining the structure and content of the sentence as opposed to word-level errors (see appendix e-1). Moreover, the authors of the recommendations noted that single word repetition can be spared in
lvPPA, implying that the ratio of sentence to single word repetition might be a more specific indicator. When we replaced sentence repetition with a ratio of sentence (of 6-8 syllables) to single 2-syllable word repetition to see whether this might tease out a logopenic type factor, this variable still aligned with the nfvPPA factor (data not shown). The proposed lvPPA criteria also stipulate phonologic errors, but this feature appeared in the fourth axis in isolation.

Taken together, the results of this prospective data-driven study raise questions about the existence of lvPPA as a discrete linguistic syndrome. The results do identify 3 groups in the sense that a substantial proportion of patients have neither the semantic nor the nonfluent/agrammatic variant. One can define a group by its absence of the features of other groups, but to call this a syndrome would not make sense. The 3 positive features proposed in lvPPA, impaired word retrieval, impaired sentence repetition, and phonologic errors, may simply be insufficiently specific to separate this group from the other 2 syndromes. This is not an unprecedented finding because a lack of specific and distinctive features in the logopenic group has previously been documented in the literature. ${ }^{26}$ The remaining criteria for lvPPA were all negatives: absence of 1) impairment in word/ object comprehension, 2) apraxia of speech, and 3) agrammatism. In other words, to some degree, implicit in the recommendations themselves is the notion that this syndrome is defined by the absence of the characteristics of the other 2 syndromes.

Previous studies have reported that, of the 3 syndromic variants, lvPPA is most associated with Alzheimer pathology $y^{27,28}$ although pathologic homogeneity cannot be viewed as evidence for a discrete clinical syndrome. Only 2 patients (4\%) met the criteria for lvPPA in this consecutive cohort, yet previous studies have suggested that Alzheimer pathology is the underlying diagnosis in almost one-third of patients with PPA. 29,30 Mixed PPA, in contrast, accounted for about one-third of cases in this cohort. We speculate that unlike the 2 reasonably discrete syndromes of semantic and nonfluent/agrammatic PPA, which are mainly associated with non-Alzheimer disease pathology, ${ }^{31,32}$ the linguistically heterogeneous features in these patients (mixed PPA) are most likely to be those associated with Alzheimer pathology.

Based on the present results, we propose that there are 2 clearly definable PPA syndromes: semantic and nonfluent/agrammatic. There are, in addition, a substantial number of patients with PPA who are not accommodated in these 2 syndromes. Such patients are heterogeneous in terms of language features. If one adopts a priori a tripartite view of PPA, as was recently proposed, then 3 groups will emerge, 
but in this scenario, one syndrome will be defined as the absence of the other 2 .

A caveat to the present findings is that this analysis was confined to the language features of the recently proposed recommendations; it is therefore at least possible that other linguistic markers may identify presently unknown language syndromes. In addition, on a methodologic note, we acknowledge that the number of participants in our study was relatively small compared with the number of language markers assessed. Conversely, the strength of the factor loadings, especially in the first 2 axes, which led to factor saturations greater than 0.6 , argues for reliability of our findings: previous studies have suggested that strong factor loadings make the analysis less dependent upon the sample size. ${ }^{33}$ Nevertheless, we acknowledge that this has been an exploratory analysis, and further studies are required to evaluate the findings of the present work.

\section{AUTHOR CONTRIBUTIONS}

Dr. Sajjadi: recruited patients, designed the neuropsychological test battery and developed one of the novel neuropsychological tests (sentence comprehension test [SECT]) under the supervision of Dr. Patterson and Dr. Nestor, collected part of the neuropsychological data, collected and analyzed the speech samples, carried out parts of the statistical analyses, prepared the first draft of Methods and Results sections, prepared the supplementary material, and finalized the manuscript in collaboration with Dr. Nestor and K. Patterson. K. Patterson: supervised Dr. Sajjadi in preparing the novel neuropsychological tests and analysis of speech samples, conceived the study in collaboration with Dr. Nestor and Dr. Sajjadi, revised and rewrote different parts of the manuscript. R.J. Arnold: collected a significant proportion of the neuropsychological data, collaborated in development of the novel neuropsychological tests. Dr. Watson: carried out major parts of the statistical analysis. Dr. Nestor: supervised Dr. Sajjadi in developing SECT, devised the make a sentence test (MAST) test, conceived the study in collaboration with Dr. Sajjadi and K. Patterson, first drafted introduction and discussion sections of the paper and revised and finalized the whole manuscript in collaboration with Dr. Sajjadi and K. Patterson.

\section{DISCLOSURE}

The authors report no disclosures relevant to the manuscript. Go to Neurology.org for full disclosures.

Received October 11, 2011. Accepted in final form January 23, 2012.

\section{REFERENCES}

1. Mesulam MM. Primary progressive aphasia. Ann Neurol 2001;49:425-432.

2. Grossman JM, Onishi K, Hughes E, et al. Progressive nonfluent aphasia: language, cognitive, and PET measures contrasted with probable Alzheimer's disease. J Cogn Neurosci 1996;8:135-154

3. Hodges JR, Patterson K, Oxbury S, Funnell E. Semantic dementia: progressive fluent aphasia with temporal lobe atrophy. Brain 1992;115:1783-1806.

4. Gorno-Tempini ML, Dronkers NF, Rankin KP, et al. Cognition and anatomy in three variants of primary progressive aphasia. Ann Neurol 2004;55:335-346.

5. Gorno-Tempini ML, Hillis AE, Weintraub S, et al. Classification of primary progressive aphasia and its variants. Neurology 2011;76:1006-1014.
6. Ash S, Moore P, Antani S, McCawley G, Work M, Grossman M. Trying to tell a tale: discourse impairments in progressive aphasia and frontotemporal dementia. Neurology 2006;66:1405-1413.

7. Wilson SM, Henry ML, Besbris M, et al. Connected speech production in three variants of primary progressive aphasia. Brain 2010;133:2069-2088.

8. Osher JE, Wicklund AH, Rademaker A, Johnson N, Weintraub $S$. The mini-mental state examination in behavioral variant frontotemporal dementia and primary progressive aphasia. Am J Alzheimers Dis Other Demen 2007;22:468-473.

9. Grossman M, Xie SX, Libon DJ, et al. Longitudinal decline in autopsy-defined frontotemporal lobar degeneration. Neurology 2008;70:2036-2045.

10. Mioshi E, Dawson K, Mitchell J, Arnold R, Hodges JR. The Addenbrooke's Cognitive Examination Revised (ACE-R): a brief cognitive test battery for dementia screening. Int J Geriatr Psychiatry 2006;21:1078-1085.

11. Swinburn K, Porter G, Howard D. Comprehensive Aphasia Test. London: Psychology Press; 2004.

12. Petersen RC, Smith GE, Waring SC, Ivnik RJ, Tangalos EG, Kokmen E. Mild cognitive impairment: clinical characterization and outcome. Arch Neurol 1999;56:303-308.

13. Field A. Discovering Statistics using SPSS. London: Sage Publications; 2009.

14. Mioshi E, Kipps CM, Dawson K, Mitchell J, Graham A, Hodges JR. Activities of daily living in frontotemporal dementia and Alzheimer disease. Neurology 2007;68:20772084.

15. Xiong L, Xuereb JH, Spillantini MG, Patterson K, Hodges JR, Nestor PJ. Clinical comparison of progressive aphasia associated with Alzheimer versus FTD-spectrum pathology. J Neurol Neurosurg Psychiatry 2011;82:254-260.

16. Mesulam M, Wieneke C, Rogalski E, Cobia D, Thompson C, Weintraub S. Quantitative template for subtyping primary progressive aphasia. Arch Neurol 2009;66:15451551.

17. Josephs KA, Duffy JR, Strand EA, et al. Clinicopathological and imaging correlates of progressive aphasia and apraxia of speech. Brain 2006;129:1385-1398.

18. Ogar JM, Dronkers NF, Brambati SM, Miller BL, GornoTempini ML. Progressive nonfluent aphasia and its characteristic motor speech deficits. Alzheimer Dis Assoc Disord 2007;21:S23-S30.

19. Ricci M, Magarelli M, Todino V, Bianchini A, Calandriello E, Tramutoli R. Progressive apraxia of speech presenting as isolated disorder of speech articulation and prosody: a case report. Neurocase 2008;14:162-168.

20. Josephs KA, Duffy JR, Fossett TR, et al. Fluorodeoxyglucose F18 positron emission tomography in progressive apraxia of speech and primary progressive aphasia variants. Arch Neurol 2010;67:596-605.

21. Croot K. Diagnosis of AOS: definition and criteria. Semin Speech Lang 2002;23:267-280.

22. Kertesz A, Davidson W, McCabe P, Takagi K, Munoz D. Primary progressive aphasia: diagnosis, varieties, evolution. J Int Neuropsychol Soc 2003;9:710-719.

23. Ogar J, Slama H, Dronkers N, Amici S, Gorno-Tempini ML. Apraxia of speech: an overview. Neurocase 2005;11: 427-432.

24. Rogalski E, Cobia D, Harrison TM, et al. Anatomy of language impairments in primary progressive aphasia. J Neurosci 2011;31:3344-3350. 
25. Ash S, McMillan C, Gunawardena D, et al. Speech errors in progressive non-fluent aphasia. Brain Lang 2010;113:13-20.

26. Hu WT, McMillan C, Libon D, et al. Multimodal predictors for Alzheimer disease in nonfluent primary progressive aphasia. Neurology 2010;75:595-602.

27. Rabinovici GD, Jagust WJ, Furst AJ, et al. A $\beta$ amyloid and glucose metabolism in three variants of primary progressive aphasia. Ann Neurol 2008;64:388-401.

28. Mesulam M, Wicklund A, Johnson N, et al. Alzheimer and frontotemporal pathology in subsets of primary progressive aphasia. Ann Neurol 2008;63:709-719.

29. Alladi S, Xuereb J, Bak T, et al. Focal cortical presentations of Alzheimer's disease. Brain 2007;130:2636-2645.
30. Mesulam MM, Grossman M, Hillis A, Kertesz A, Weintraub $S$. The core and halo of primary progressive aphasia and semantic dementia. Ann Neurol 2003;54(suppl 5): S11-S14.

31. Snowden J, Neary D, Mann D. Frontotemporal lobar degeneration: clinical and pathological relationships. Acta Neuropathol 2007;114:31-38.

32. Hodges JR, Mitchell J, Dawson K, et al. Semantic dementia: demography, familial factors and survival in a consecutive series of 100 cases. Brain 2010;133:300-306.

33. Guadagnoli E, Velicer WF. Relation of sample size to the stability of component patterns. Psychol Bull 1988;103: $265-275$.

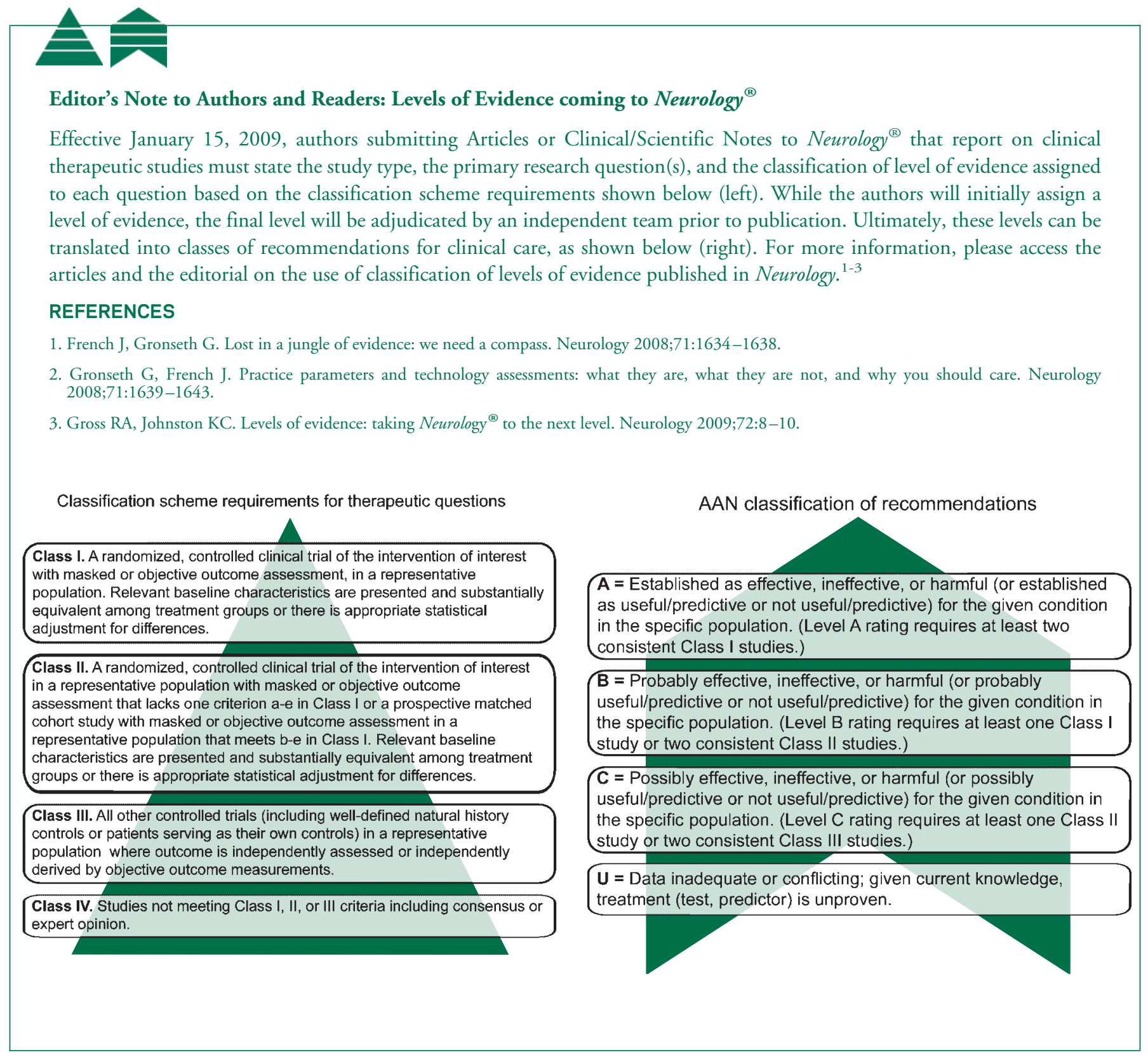

